Nguyen, N., Van Hertem, D., Tielens, P., Driesen, J. (2012). Damping Controller Design of TCSC Based on Wide-Area Monitoring and Supplement Torque Components. . Energycon 2012. Florence, Italy, 9-12 September 2012.

Digital Object Identifier: 10.1109/EnergyCon.2012.6347791

URL:

http://ieeexplore $\cdot$ ieee. org/stamp/stamp $\cdot$ jsp?tp=\&arnumber=6347791

(C) 2012 IEEE. Personal use of this material is permitted. Permission from IEEE must be obtained for all other users, including reprinting/ republishing this material for advertising or promotional purposes, creating new collective works for resale or redistribution to servers or lists, or reuse of any copyrighted components of this work in other works. 


\title{
Damping Controller Design of TCSC Based on Wide-Area Monitoring and Supplement Torque Components
}

\author{
Nguyen Tuan Anh, Dirk Van Hertem, Senior Member, IEEE, \\ Pieter Tielens, Student Member, IEEE, and Johan Driesen, Senior Member, IEEE \\ Electrical engineering department \\ Research group ELECTA \\ KU Leuven, Belgium \\ Contact: Anh.NguyenTuan@esat.kuleuven.be, Dirk.VanHertem@esat.kuleuven.be, \\ Pieter.Tielens@esat.kuleuven.be, Johan.Driesen@esat.kuleuven.be
}

\begin{abstract}
In this paper, the effectiveness of a Thyristor Controlled Series Capacitor (TCSC) using wide-area measurements to damp oscillations is considered. Based on a small-signal stability analysis of a single-machine infinite bus system with TCSC, a detailed theoretical framework is developed to design a Power Oscillation Damping (POD) controller modulated by a remote input signal. Phasor Measurement Units (PMUs) are used to measure real-time remote signals and transfer them to the TCSC stability control loop. The optimal controller design of the TCSC based on the supplement torque components is determined. Test cases show the improvement of the small-signal stability by using a remote control signal instead of a local one. Furthermore, the results demonstrate the practical application capability of wide-area measurements for the improvement of power system stability.
\end{abstract}

Index Terms-FACTS, oscillation damping, PMU, remote input signal, single-machine infinite bus system, TCSC design

\section{INTRODUCTION}

$\mathbf{P}$ RACTICAL interconnected power systems have numerous modes of electro-mechanical oscillations. These oscillation modes normally occur in the frequency range of 0.1 to $2 \mathrm{~Hz}$, and can be placed into two categories: local modes and inter-area modes [1], [2]. If a disturbance occurs and the oscillations are not well damped, they may continue to grow and cause instability and loses of synchronism in the whole power system. The traditional method used to increase the damping of a power system is adding a Power System Stabilizer (PSS) to the excitation system of a generator. The PSS, with the speed deviation as an input signal, introduces a damping torque component. The stabilizer typically has a great performance with respect to the local oscillation modes, but often fails to effectively deal with the inter-area oscillation modes [3]. With changes in power system structure as well as system operating conditions, the tuning of the exciter and PSS may not be flexible enough to deliver sufficient damping and synchronizing torque components to damp the oscillations. Thus, new controls and system elements are needed to provide the supplement torque components to maintain a secure system operation.

Thyristor Controlled Series Capacitor (TCSC), a prominent Flexible AC Transmission System (FACTS) device, offers a great potential in damping power system oscillations [4], [5]. In literature, three problems regarding the damping of oscillations by TCSCs have been frequently addressed: effective input signals, placement of TCSC and optimal design of the Power Oscillation Damping (POD) controller.

Local as well as remote signals can be employed as an input signal for modulating the TCSC [5]. Due to measurement and communication difficulties, most applications select local variables as input signals. However, only a limited number of signals are locally available, and this might limit the effectiveness of the controller. Furthermore, it has been shown that when remote signals are fed to the controller, the system dynamic performance can be enhanced compared to using locally measured signals [6].

Technological developments in the Wide-Area Measurement System (WAMS) allow a centralized control for improving the power system stability. Measurements by Phasor Measurement Units (PMUs) have reached the point where it is possible to use them for wide scale applications in large power systems [7], [8]. Advantages of the WAMS include using the real-time remote signals measured by PMUs as an input for the FACTS controller to damp the oscillations.

In practical applications, where a TCSC is installed in a tie-line connecting two areas, the controller location has been chosen to meet the requirements to enhance transfer capability or to improve power exchange. Hence, the series compensation level has been defined for a certain steady-state operating condition.

The POD design on the other hand focuses mainly on determining a phase compensation network and a gain to provide sufficient additional torque components. The design of this damping controller is generally based on small-signal analysis using linear techniques, which provides valuable information about the dynamic characteristics of the closed-loop control system in the desired frequency range of the swing modes [4]. The functional sensitivity, or the residue method, based on this linear technique, has been proven successfully in designing the POD controllers [3], [9]-[11]. However, residue analysis requires an advanced linear model of the entire system. In a very large inter-connected power system, 
this method is limited by the available computational power and the difficulties in detecting the dominant eigenvalues.

This paper proposes a design method for the TCSC damping controller based on the synchronized phasor measurements and additional torque components. The paper is organized as follows: in section II, the small-signal stability analysis of a single-machine infinite bus system with a TCSC as shown in Fig. 1 is described to provide a better insight and understanding of the basic properties of the damping effect from the controller. Section III deals with the design method for the POD controller using a remote input (RI) signal. The design is based on the analyses of the damping torque components provided by the TCSC. Next, in section IV the applicability of this design method in a multi-machine system is discussed. The effectiveness of the designs based on remote and local input signals are compared in Section V. Section VI draws the main conclusions.

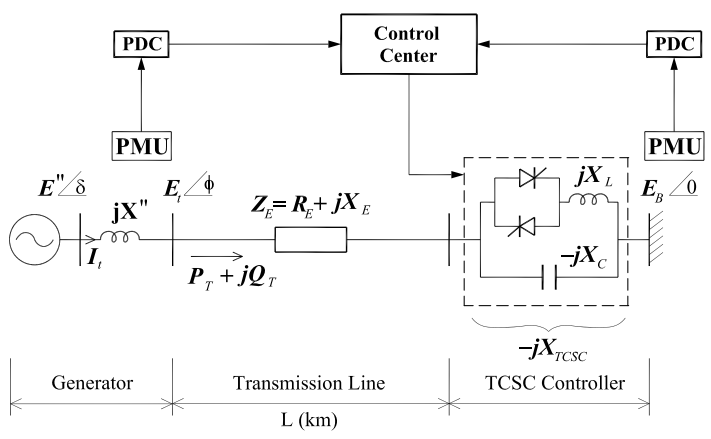

Fig. 1. Single-machine infinite bus system with a TCSC

\section{Small-Signal Stability AnALYSiS}

It is assumed that a TCSC controller is installed in a long distance tie-line of a multi-machine power system. The location and compensating level of the TCSC were primarily selected based on the original objective of increasing the power exchange between two areas. In other words, the desired steady-state series compensation level, or the reactance reference of the TCSC was determined by the steady-state power flow solution. In this case, the optimal POD design of the TCSC for damping power oscillations is considered. From the point of view of the POD controller design, the multi-machine system external to the generator and the TCSC may be reduced to a Thévenin's equivalent as shown in Fig. 1. This system model consists of one synchronous generator connected to an infinite-bus system through a tie-line compensated by a TCSC. The generator may represent one or a group of large power plants injecting the scheduled power to the system via the TCSC. In Fig. $1, Z_{E}$ is the impedance of the transmission line as seen from the TCSC.

The rotor angle deviation of this remote generator is chosen as an input signal for the POD controller. Since the rotor angle can not be measured directly by the PMU, it is taken proportional to the phase angle of the generator terminal voltage. As shown in Fig. 1, two PMUs are installed, one at the generator location and one at the infinite bus, providing each a synchronized phasor measurement. Both signals are transmitted to the Phasor Data Concentrator (PDC). The PDC is used to synchronize the measurements of PMUs and send the data every $20 \mathrm{~ms}$ to the control center [12]. The WAMS precise time synchronization is accomplished and the data is sent to the TCSC location.

\section{A. Synchronous Generator Model}

In order to study the detailed dynamic interaction between the generator and the TCSC, a sixth order salientpole synchronous generator model is employed to include the effects of the dynamics of the field circuits, excitation system and damper windings, one in the $d$-axis and two in the $q$-axis. A thyristor based exciter is used as excitation system. An Automatic Voltage Regulator (AVR) and a Power System Stabilizer (PSS) are also added. The turbine-governor model is on the other hand ignored. The complete equations representing the generator and network models are given in [2].

\section{B. TCSC Model}

The TCSC dynamics during power-swing frequencies are modeled by a variable reactance at fundamental frequency. The reactance value is generated from the POD controller which is a pre-programmed open-loop controller to damp power oscillations. The POD structure is shown in Fig. 2. Basically, it consists of a static gain $K_{C}$, which is adjusted to obtain the desired damping; a washout block defined by a time constant $T_{w}$ and the dynamic compensator defined by the time constants $T_{1}, T_{2}, T_{3}$ and $T_{4}$. The main use of the washout filter is to avoid the controller to response to the dc offset of the input signal. The dynamic compensator consists of one or more leadlag blocks to provide the necessary phase-lag characteristics. The desired magnitude of the TCSC reactance, $\Delta X_{T C S C}$, is obtained after a small time delay caused by the firing controls and the natural response of the TCSC. This delay is modelled by a lag circuit having a time constant, $T$, of typically $15-20$ $\mathrm{ms}$ [5]. The input signal that can be employed for modulating the TCSC reactance is the rotor-angle deviation of the remote generator, $\Delta \delta$.

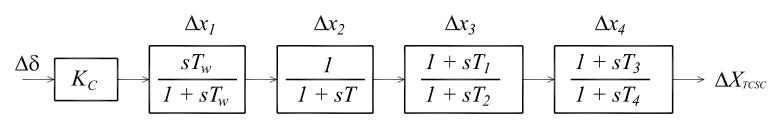

Fig. 2. POD controller of TCSC

In the small signal stability analysis, to model the POD controller of the TCSC, $\Delta x_{1}, \Delta x_{2}, \Delta x_{3}$ and $\Delta x_{4}$ are chosen as additional state variables of the system.

\section{Block Diagram Representation of System with TCSC}

For the small signal stability analysis, it is required that all the state equations representing the generator and dynamic devices such as TCSC controller are linearized. Since the state 


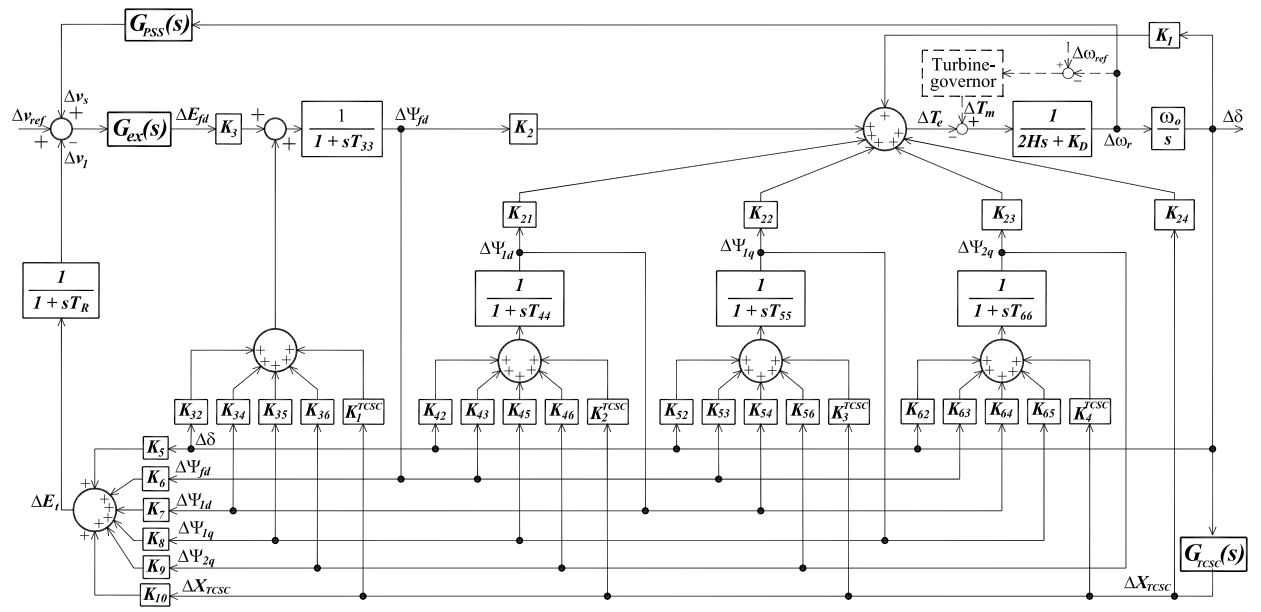

Fig. 3. Block diagram representation of system with TCSC

matrix of the entire system is established, the system model can be expressed by a block diagram representation as shown in Fig. 3.

In this figure, $G_{e x}(s)$ is the transfer function of the exciter, $G_{P S S}(s)$ is the transfer function of the PSS and $G_{T C S C}(s)$ is the transfer function of the POD controller. $K_{1}, K_{2}, K_{21}, K_{22}$, $K_{23}$ and $K_{24}$ are the constants used to express the electrical torque equation $\left(\Delta T_{e}\right) . K_{5}$ to $K_{10}$ are the constants for the expression of the generator terminal voltage $\left(\Delta E_{t}\right) . T_{33}, K_{3}$, $K_{32}, K_{34}, K_{35}, K_{36}$ and $K_{1}^{T C S C}$ are the constants used to describe the field flux linkage $\left(\Delta \psi_{f d}\right) . T_{44}, K_{42}, K_{43}, K_{45}$, $K_{46}$ and $K_{2}^{T C S C}$ are the constants used to describe the $d$-axis amortisseur flux linkage $\left(\Delta \psi_{1 d}\right)$. Finally $T_{55}, T_{66}, K_{52}, K_{62}$, $K_{53}, K_{63}, K_{54}, K_{64}, K_{56}, K_{65}, K_{3}^{T C S C}$ and $K_{4}^{T C S C}$ are the constants used to describe the $q$-axis amortisseurs flux linkages $\left(\Delta \psi_{1 q}\right.$ and $\left.\Delta \psi_{2 q}\right)$. The expressions for all the constants in the block diagram of Fig. 3 can be found in the Appendix A.

From the block diagram follows that the electromagnetic torque contribution of the TCSC damping controller is through blocks $K_{10}, K_{1}^{T C S C}, K_{2}^{T C S C}, K_{3}^{T C S C}, K_{4}^{T C S C}$ and $K_{24}$. Fig. 3 also shows that only the contribution through $K_{24}$ is direct, while the contributions through other blocks are attenuated by the first-order lag blocks. By examining the block diagram and the expressions of the constants, with the TCSC reactance modulation, the greatest damping torque contribution by the controller is a channel through block $K_{10}$ to the exciter which modifies the field voltage.

The system model of Fig. 3 is also described by a closedloop transfer function as shown in Fig. 4. It is used to express the contribution of the different torque components to the rotor angle deviation.

In Fig. 4, $\Delta T_{e}^{t c s c}$ is the supplement damping torque generated by the TCSC. $\Delta T_{e}^{e x}$ is the total of the synchronizing and damping torque components of the generator. $G_{T C S C}(s)$ is the transfer function of the POD controller as shown in Fig. 2. The block diagram from Fig. 3 is reduced to only include

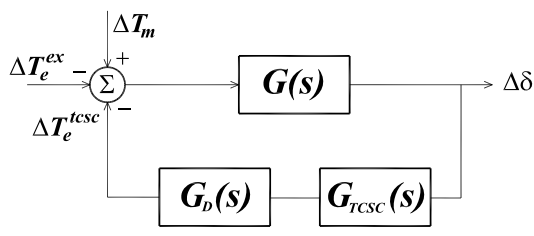

Fig. 4. Transfer function of system with TCSC

the damping components of the TCSC. A transfer function $G_{D}(s)$ is derived:

$$
\begin{aligned}
& G_{D}(s)=K_{24}+K_{4}^{T C S C} \cdot K_{23} \cdot \frac{1}{1+s T_{66}} \\
& +K_{3}^{T C S C} \cdot K_{22} \cdot \frac{1}{1+s T_{55}}+K_{2}^{T C S C} \cdot K_{21} \cdot \frac{1}{1+s T_{44}} \\
& +K_{2} \cdot \frac{1}{1+s T_{33}} \cdot\left(K_{1}^{T C S C}-K_{3} \cdot K_{10} \cdot G_{e x} \cdot \frac{1}{1+s T_{R}}\right)
\end{aligned}
$$

\section{Damping Controller Design Method}

Small signal stability analysis of the system without TCSC needs to be carried out to identify the electromagnetic torque $\Delta T_{e}^{e x}$. This torque includes two components: synchronizing and damping torque. The damping torque depends mainly on the performance capability of the excitation system along with the PSS. The TCSC can supply additional synchronizing and damping torque components for damping oscillations in a given frequency range. These torque components can be expressed in a complex form as follows:

$$
\Delta T_{e}^{t c s c}=\left(K_{\text {syn }}+j K_{d a m}\right) \Delta \delta=K \angle \beta
$$

where $K_{\text {syn }}$ and $K_{d a m}$ are the synchronizing and damping torque coefficients, respectively. $K$ and $\beta$ are the magnitude and phase angle of $\Delta T_{e}^{t c s c}$.

The design of the POD controller (Fig. 2) includes the determination of the lead-lag compensator and the gain. It is 
assumed that the system has a critical oscillation mode with frequency $\omega$. To sufficiently damp this mode, the following equations should be satisfied:

$$
\begin{aligned}
\beta & =\arg \left[G_{D}(s)\right]+\arg \left[G_{T C S C}(s)\right] \\
K & =\left|G_{D}(s)\right| \cdot\left|G_{T C S C}(s)\right|
\end{aligned}
$$

From Equations (3) and (4), the phase angle and the magnitude of the POD controller transfer function, $G_{T C S C}(s)$ can be defined. The magnitude and phase angle of the transfer function $G_{D}(s)$ can be determined by examining the frequency response corresponding to the oscillation frequency $\omega$. Hence, the procedure of designing the POD controller is proposed as follows:

$$
G_{T C S C}(s)=K_{C} \cdot \frac{s T_{w}}{1+s T_{w}} \cdot \frac{1}{1+s T} \cdot \frac{1+s T_{1}}{1+s T_{2}} \cdot \frac{1+s T_{3}}{1+s T_{4}}
$$

with:

$$
\begin{aligned}
& \phi=\beta-\arg \left[G_{D}(s)\right]-\arg \left[\frac{s T_{w}}{1+s T_{w}}\right]-\arg \left[\frac{1}{1+s T}\right] \\
& \alpha=\frac{T_{2}}{T_{1}}=\frac{T_{4}}{T_{3}}=\frac{1-\sin \left(\frac{\phi}{2}\right)}{1+\sin \left(\frac{\phi}{2}\right)} \\
& T_{1}=T_{3}=\frac{1}{\omega \sqrt{\alpha}} \\
& T_{2}=T_{4}=\alpha T_{1}
\end{aligned}
$$

where $T_{w}$ is the washout time constant; $T$ is the total time delay; $\omega$ is frequency of the poorly damped mode in $\mathrm{rad} / \mathrm{sec}$; and $K_{C}$ is a positive constant gain.

Since the time constants $T_{1}, T_{2}, T_{3}$ and $T_{4}$ are set, the gain of the POD controller is determined as follows:

$$
K_{C}=\frac{K /\left|G_{D}(s)\right|}{\left|\frac{s T_{w}}{1+s T_{w}} \cdot \frac{1}{1+s T} \cdot \frac{1+s T_{1}}{1+s T_{2}} \cdot \frac{1+s T_{3}}{1+s T_{4}}\right|}
$$

IV. Applicability of Design Method in a Multi-Machine System With Communication Time DELAY

Power systems are regularly expanding to meet the increasing load demand and to ensure a secure system operation. New generating units and power plants require long transmission links to inject the scheduled power into the system. In this case, TCSCs are installed in order to improve transfer capability and to damp power oscillations. Since the new generating units or groups may be reduced to an equivalent generator inter-connected to the rest of the system through the TCSC and the long tie-line, the proposed design method for the TCSC damping controller can be applied in multi-machine systems. The computational effort is significantly reduced as compared to the traditional design methods, which have to take into account the detailed models of the entire system.

To consider the practical application of remote measurements, it is a necessary to discuss the communication delay of PMU/WAMS system. Communication links employed in
WAMS include both wired (telephone lines and fiber-optics) as wireless (satellites and microwave links) options. The total delay of delivering the synchronizing signal from the PMUs to the TCSC location is the latency of the slowest line plus the time needed for synchronization. References [13], [14] indicate that the delay using optical fiber communication in a PMU/WAMS system, is typically 40 - $60 \mathrm{~ms}$. So, together with the time for synchronization, the total transmission delay is about $80-120 \mathrm{~ms}$. It is necessary to take this delay into account to investigate the effectiveness of the design method proposed before.

Wide-area measurements are rarely free from noise. The noises can be eliminated by using the effective filters and higher sampling rates in PMUs. The fiber-optic communication links are a suitable option to reduce the effects of noise [15].

\section{Test CAses}

This section demonstrates the performance of the TCSC designs using wide-area measurements for damping power oscillations. The parameters of the test system (Fig. 1) are given in Appendix B.

A small signal stability analysis of the system is carried out with the length of the tie-line varying from $120 \mathrm{~km}$ to 600 $\mathrm{km}$ (without TCSC control). By scanning the rotor angle/speed oscillation frequencies in the range from $0.1 \mathrm{~Hz}$ to $2.0 \mathrm{~Hz}$, the critical modes are detected and shown in Table I. It is seen that, these modes are stable and have a small damping ratio.

Applying the design method proposed in Section III, the optimal parameters of the POD controller using RI (rotor angle deviation) and LI (active power flowing through the TCSC) signals have been computed. For the two TCSC designs corresponding RI and LI signals, the desired supplement damping torque is given by $\Delta T_{e}^{t c s c}=0.05 \angle 90^{0}$, i.e. a pure damping torque. The total time delay $T$ for remote and local input signals of the controller are assumed to be $200 \mathrm{~ms}$ (including the communication delay) and $50 \mathrm{~ms}$, respectively. The washout time constant $T_{w}$ for both local and remote measurement is 1.5 seconds.

TABLE I

OSCILlation Modes - Without TCSC CONTROL

\begin{tabular}{|c|c|c|c|}
\hline $\begin{array}{c}\text { Length } \\
(\mathrm{km})\end{array}$ & Eigenvalues & $\begin{array}{c}\omega_{d} \\
(\mathrm{~Hz})\end{array}$ & $\begin{array}{c}\text { Damping } \\
\text { ratio }\end{array}$ \\
\hline 120 & $-0.900 \pm j 9.387$ & 1.494 & 0.095 \\
\hline 180 & $-0.575 \pm j 8.340$ & 1.327 & 0.069 \\
\hline 240 & $-0.398 \pm j 7.547$ & 1.201 & 0.053 \\
\hline 300 & $-0.291 \pm j 6.905$ & 1.099 & 0.042 \\
\hline 360 & $-0.222 \pm j 6.358$ & 1.012 & 0.035 \\
\hline 420 & $-0.176 \pm j 5.870$ & 0.934 & 0.030 \\
\hline 480 & $-0.143 \pm j 5.413$ & 0.862 & 0.026 \\
\hline 540 & $-0.118 \pm j 4.961$ & 0.790 & 0.024 \\
\hline 600 & $-0.100 \pm j 4.469$ & 0.711 & 0.022 \\
\hline
\end{tabular}


TABLE II

Optimal Design of TCSC with Remote Measurements

\begin{tabular}{|c|c|c|c|c|c|}
\hline $\begin{array}{c}\text { Length } \\
(\mathrm{km})\end{array}$ & $K_{C}$ & $\begin{array}{c}T_{1} \\
\& T_{3}\end{array}$ & $\begin{array}{c}T_{2} \\
\& T_{4}\end{array}$ & $\begin{array}{c}\text { Actual eigenvalues } \\
\left(\lambda_{i}^{r}\right)\end{array}$ & $\begin{array}{c}\text { Damping } \\
\text { ratio }\end{array}$ \\
\hline 120 & 0.24 & 0.076 & 0.149 & $-1.030 \pm j 9.408$ & 0.109 \\
\hline 180 & 0.29 & 0.085 & 0.169 & $-0.720 \pm j 8.356$ & 0.086 \\
\hline 240 & 0.34 & 0.093 & 0.189 & $-0.556 \pm j 7.560$ & 0.073 \\
\hline 300 & 0.38 & 0.100 & 0.209 & $-0.463 \pm j 6.915$ & 0.067 \\
\hline 360 & 0.43 & 0.107 & 0.230 & $-0.409 \pm j 6.366$ & 0.064 \\
\hline 420 & 0.48 & 0.115 & 0.253 & $-0.377 \pm j 5.877$ & 0.064 \\
\hline 480 & 0.53 & 0.122 & 0.279 & $-0.361 \pm j 5.419$ & 0.066 \\
\hline 540 & 0.58 & 0.131 & 0.310 & $-0.357 \pm j 4.965$ & 0.072 \\
\hline 600 & 0.63 & 0.142 & 0.352 & $-0.366 \pm j 4.472$ & 0.082 \\
\hline
\end{tabular}

TABLE III

Optimal Design of TCSC with Local Measurements

\begin{tabular}{|c|c|c|c|c|c|}
\hline $\begin{array}{c}\text { Length } \\
(\mathrm{km})\end{array}$ & $K_{C}$ & $\begin{array}{c}T_{1} \\
\& T_{3}\end{array}$ & $\begin{array}{c}T_{2} \\
\& T_{4}\end{array}$ & $\begin{array}{c}\text { Actual eigenvalues } \\
\left(\lambda_{i}^{l}\right)\end{array}$ & $\begin{array}{c}\text { Damping } \\
\text { ratio }\end{array}$ \\
\hline 120 & 0.09 & 0.063 & 0.181 & $-1.032 \pm j 9.348$ & 0.110 \\
\hline 180 & 0.17 & 0.070 & 0.204 & $-0.716 \pm j 8.292$ & 0.086 \\
\hline 240 & 0.26 & 0.078 & 0.226 & $-0.548 \pm j 7.489$ & 0.073 \\
\hline 300 & 0.39 & 0.084 & 0.248 & $-0.451 \pm j 6.837$ & 0.066 \\
\hline 360 & 0.57 & 0.091 & 0.270 & $-0.391 \pm j 6.279$ & 0.062 \\
\hline 420 & 0.81 & 0.099 & 0.294 & $-0.353 \pm j 5.778$ & 0.061 \\
\hline 480 & 1.16 & 0.107 & 0.319 & $-0.328 \pm j 5.305$ & 0.062 \\
\hline 540 & 1.74 & 0.117 & 0.349 & $-0.309 \pm j 4.832$ & 0.064 \\
\hline 600 & 2.94 & 0.129 & 0.388 & $-0.286 \pm j 4.307$ & 0.066 \\
\hline
\end{tabular}

With the modulation of the TCSC having a fixed series compensated level of $10 \%$ of the $600-\mathrm{km}$ tie-line, i.e. $X_{T C S C}^{0}=$ 0.15 p.u., small-signal stability analyses are carried out with the tie-line length varying from $120 \mathrm{~km}$ to $600 \mathrm{~km}$. The results are shown in Table II and III. The eigenvalues and damping ratios, indicate the effectiveness of the TCSC modulation to damp the oscillations. Fig. 5 shows that with the optimal design of the TCSC, the system becomes more stable since the actual eigenvalues are shifted more towards the left on the complex plane. As the tie-line length (equivalent impedance of the transmission network) increases, the damping of the

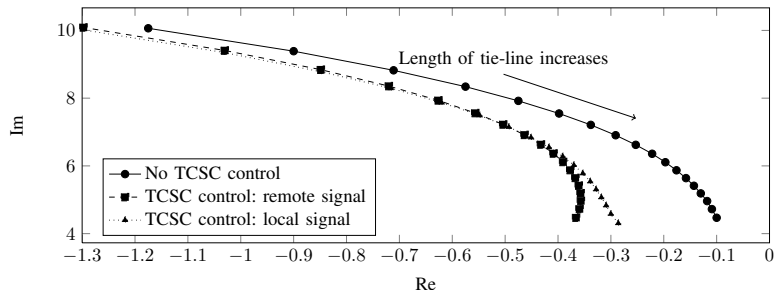

Fig. 5. Shift of eigenvalues with TCSC modulation and variation of tie-line length system is much more improved by the TCSC using the RI signal compared to using a LI signal.

The block diagram of Fig. 3 is also implemented in Matlab/Simulink. Simulations are carried out to demonstrate the damping of the TCSC, modulated both by a RI as well as a LI signal. A disturbance to the mechanical torque of $0.001 \mathrm{pu}$ is applied at the generator at $t=1 \mathrm{~s}$. The results for a tie-line of $600 \mathrm{~km}$ and $240 \mathrm{~km}$ are given in Fig. 6 and Fig. 7. It is clear that for the $600 \mathrm{~km}$ line the damping ratio is much lower than for the $240 \mathrm{~km}$ line. The results also confirm the conclusions from the eigenvalue analysis: the TCSC modulated by a RI signal provides better damping than when modulated by a LI signal.
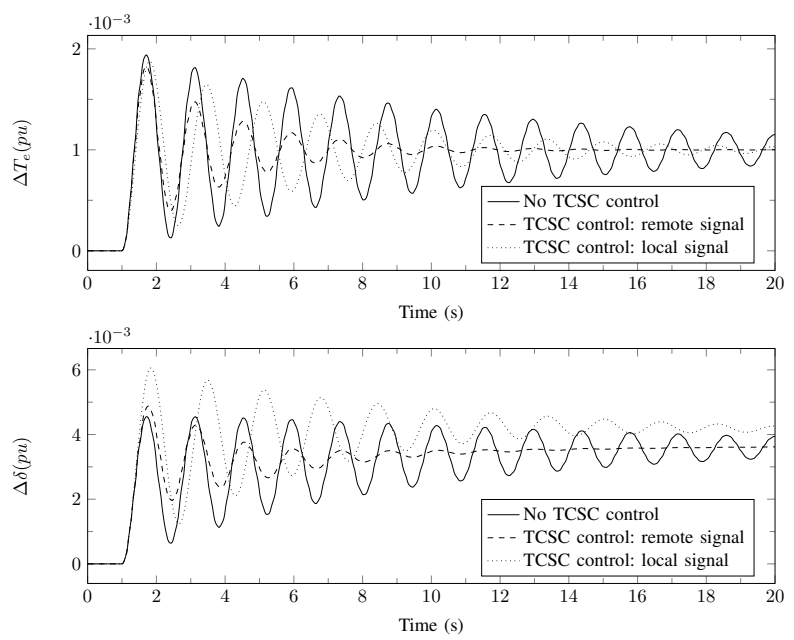

Fig. 6. Variation of $\Delta T_{e}$ and $\Delta \delta$ after a disturbance of $\Delta T_{m}=0.001 p u$ at $t=1 \mathrm{~s}$ for tie-line length of $600 \mathrm{~km}$
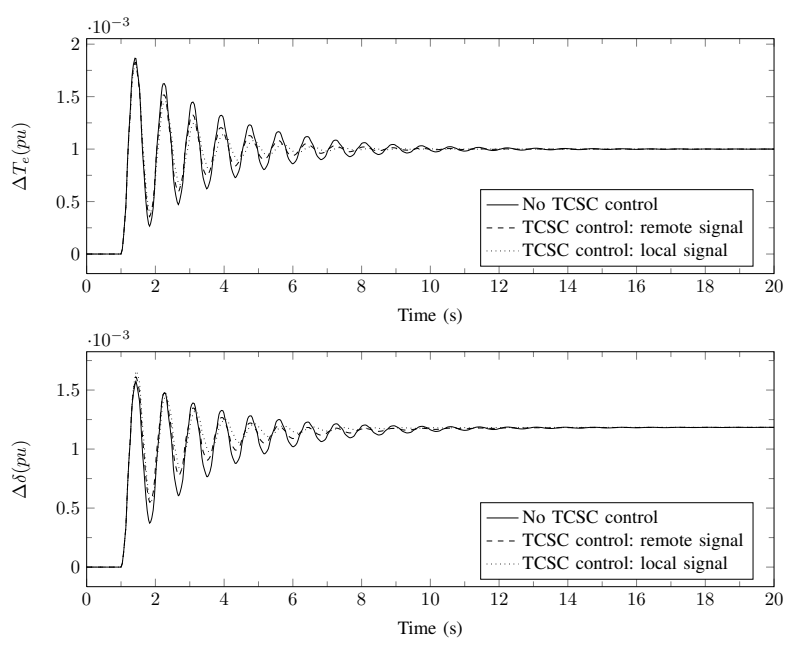

Fig. 7. Variation of $\Delta T_{e}$ and $\Delta \delta$ after a disturbance of $\Delta T_{m}=0.001 p u$ at $t=1 \mathrm{~s}$ for tie-line length of $240 \mathrm{~km}$ 


\section{CONCLUSION}

This paper has investigated the potential of a TCSC using a wide-area measurement as input signal for damping oscillations in a single-machine infinite bus system. Based on small-signal stability and supplement damping torque analysis, a design method for the POD controller of the TCSC has been proposed. The optimal controller designs have been demonstrated in the test cases. It is proven that the oscillation damping by the TCSC using the effective remote signal is better than the controller design based on local signal. The paper has also shown that when the equivalent impedance of the network increases, the remote input signal for TCSC modulation is the more suitable choice.

\section{APPENDIX A}

EXPRESSIONS FOR THE DIFFERENT CONSTANTS IN THE BLOCK DIAGRAM

Constants for $\Delta T_{e}$ :

$$
\begin{aligned}
K_{1} & =n_{1}\left(\psi_{a d 0}+L_{a q s}^{\prime \prime} i_{d 0}\right)-m_{1}\left(\psi_{a q 0}+L_{a d s}^{\prime \prime} i_{q 0}\right) \\
K_{2} & =n_{2}\left(\psi_{a d 0}+L_{a q s}^{\prime \prime} i_{d 0}\right)-m_{2}\left(\psi_{a q 0}+L_{a d s}^{\prime \prime} i_{q 0}\right)+\frac{L_{a d s}^{\prime \prime}}{L_{f d}} i_{q 0} \\
K_{21} & =n_{3}\left(\psi_{a d 0}+L_{a q s}^{\prime \prime} i_{d 0}\right)-m_{3}\left(\psi_{a q 0}+L_{a d s}^{\prime \prime} i_{q 0}\right)+\frac{L_{a d s}^{\prime \prime}}{L_{1 d}} i_{q 0} \\
K_{22} & =n_{4}\left(\psi_{a d 0}+L_{a q s}^{\prime \prime} i_{d 0}\right)-m_{4}\left(\psi_{a q 0}+L_{a d s}^{\prime \prime} i_{q 0}\right)-\frac{L_{a q s}^{\prime \prime}}{L_{1 q}} i_{d 0} \\
K_{23} & =n_{5}\left(\psi_{a d 0}+L_{a q s}^{\prime \prime} i_{d 0}\right)-m_{5}\left(\psi_{a q 0}+L_{a d s}^{\prime \prime} i_{q 0}\right)-\frac{L_{a q s}^{\prime \prime}}{L_{2 q}} i_{d 0} \\
K_{24} & =n_{6}\left(\psi_{a d 0}+L_{a q s}^{\prime \prime} i_{d 0}\right)-m_{6}\left(\psi_{a q 0}+L_{a d s}^{\prime \prime} i_{q 0}\right)
\end{aligned}
$$

Constants for $\Delta E_{t}$

$$
\begin{aligned}
K_{5}= & \frac{e_{d 0}}{E_{t 0}}\left(-R_{a} m_{1}+n_{1} L_{l}+m_{1} L_{a d s}^{\prime \prime}\right) \\
& +\frac{e_{q 0}}{E_{t 0}}\left(-R_{a} n_{1}-m_{1} L_{l}-n_{1} L_{a q s}^{\prime \prime}\right) \\
K_{6}= & \frac{e_{d 0}}{E_{t 0}}\left[-R_{a} m_{2}+n_{2} L_{l}-L_{a d s}^{\prime \prime}\left(\frac{1}{L_{f d}}-m_{2}\right)\right] \\
& +\frac{e_{q 0}}{E_{t 0}}\left(-R_{a} n_{2}-m_{2} L_{l}-n_{2} L_{a q s}^{\prime \prime}\right) \\
K_{7}= & \frac{e_{d 0}}{E_{t 0}}\left[-R_{a} m_{3}+n_{3} L_{l}-L_{a d s}^{\prime \prime}\left(\frac{1}{L_{1 d}}-m_{3}\right)\right] \\
& +\frac{e_{q 0}}{E_{t 0}}\left(-R_{a} n_{3}-m_{3} L_{l}-n_{3} L_{a q s}^{\prime \prime}\right) \\
K_{8}= & \frac{e_{d 0}}{E_{t 0}}\left(-R_{a} m_{4}+n_{4} L_{l}+m_{4} L_{a d s}^{\prime \prime}\right) \\
& +\frac{e_{q 0}}{E_{t 0}}\left[-R_{a} n_{4}-m_{4} L_{l}+L_{a q s}^{\prime \prime}\left(\frac{1}{L_{1 q}}-n_{4}\right)\right] \\
K_{9}= & \frac{e_{d 0}}{E_{t 0}}\left(-R_{a} m_{5}+n_{5} L_{l}+m_{5} L_{a d s}^{\prime \prime}\right) \\
& +\frac{e_{q 0}}{E_{t 0}}\left[-R_{a} n_{5}-m_{5} L_{l}+L_{a q s}^{\prime \prime}\left(\frac{1}{L_{2 q}}-n_{5}\right)\right] \\
K_{10}= & \frac{e_{d 0}}{E_{t 0}}\left(-R_{a} m_{6}+n_{6} L_{l}+m_{6} L_{a d s}^{\prime \prime}\right) \\
& +\frac{e_{q 0}}{E_{t 0}}\left(-R_{a} n_{6}-m_{6} L_{l}-n_{6} L_{a q s}^{\prime \prime}\right)
\end{aligned}
$$

Constants for $\Delta \psi_{f d}$

$$
\begin{aligned}
T_{33} & =\frac{L_{f d}^{2}}{\omega_{0} R_{f d}\left(L_{a d s}^{\prime \prime} m_{2} L_{f d}+L_{f d}-L_{a d s}^{\prime \prime}\right)} \\
K_{3} & =\frac{L_{f d}^{2}}{L_{a d u}\left(L_{a d s}^{\prime \prime} m_{2} L_{f d}+L_{f d}-L_{a d s}^{\prime \prime}\right)} \\
K_{32} & =\frac{L_{a d s}^{\prime \prime} L_{f d}}{L_{a d s}^{\prime \prime}-L_{a d s}^{\prime \prime} m_{2} L_{f d}-L_{f d}} m_{1} \\
K_{34} & =\frac{L_{a d s}^{\prime \prime} L_{f d}}{L_{a d s}^{\prime \prime}-L_{a d s}^{\prime \prime} m_{2} L_{f d}-L_{f d}}\left(m_{3}-\frac{1}{L_{1 d}}\right) \\
K_{35} & =\frac{L_{a d s}^{\prime \prime} L_{f d}}{L_{a d s}^{\prime \prime}-L_{a d s}^{\prime \prime} m_{2} L_{f d}-L_{f d}} m_{4} \\
K_{36} & =\frac{L_{a d s}^{\prime \prime} L_{f d}}{L_{a d s}^{\prime \prime}-L_{a d s}^{\prime \prime} m_{2} L_{f d}-L_{f d}} m_{5} \\
K_{1}^{T C S C} & =\frac{L_{a d s}^{\prime \prime} L_{f d}}{L_{a d s}^{\prime \prime}-L_{a d s}^{\prime \prime} m_{2} L_{f d}-L_{f d}} m_{6}
\end{aligned}
$$

Constants for $\Delta \psi_{1 d}$

$$
\begin{aligned}
T_{44} & =\frac{L_{1 d}^{2}}{\omega_{0} R_{1 d}\left(L_{a d s}^{\prime \prime} m_{3} L_{1 d}+L_{1 d}-L_{a d s}^{\prime \prime}\right)} \\
K_{42} & =\frac{L_{a d s}^{\prime \prime} L_{1 d}}{L_{a d s}^{\prime \prime}-L_{a d s}^{\prime \prime} m_{3} L_{1 d}-L_{1 d}} m_{1} \\
K_{43} & =\frac{L_{a d s}^{\prime \prime} L_{1 d}}{L_{a d s}^{\prime \prime}-L_{a d s}^{\prime \prime} m_{3} L_{1 d}-L_{1 d}}\left(m_{2}-\frac{1}{L_{f d}}\right) \\
K_{45} & =\frac{L_{a d s}^{\prime \prime} L_{1 d}}{L_{a d s}^{\prime \prime}-L_{a d s}^{\prime \prime} m_{3} L_{1 d}-L_{1 d}} m_{4} \\
K_{46} & =\frac{L_{a d s}^{\prime \prime} L_{1 d}}{L_{a d s}^{\prime \prime}-L_{a d s}^{\prime \prime} m_{3} L_{1 d}-L_{1 d}} m_{5} \\
K_{2}^{T C S C} & =\frac{L_{a d s}^{\prime \prime} L_{1 d}}{L_{a d s}^{\prime \prime}-L_{a d s}^{\prime \prime} m_{3} L_{1 d}-L_{1 d}} m_{6}
\end{aligned}
$$

Constants for $\Delta \psi_{1 q}$

$$
\begin{aligned}
T_{55} & =\frac{L_{1 q}^{2}}{\omega_{0} R_{1 q}\left(L_{a q s}^{\prime \prime} n_{4} L_{1 q}+L_{1 q}-L_{a q s}^{\prime \prime}\right)} \\
K_{52} & =\frac{L_{a q s}^{\prime \prime} L_{1 q}}{L_{a q s}^{\prime \prime}-L_{a q s}^{\prime \prime} n_{4} L_{1 q}-L_{1 q}} n_{1} \\
K_{53} & =\frac{L_{a q s}^{\prime \prime} L_{1 q}}{L_{a q s}^{\prime \prime}-L_{a q s}^{\prime \prime} n_{4} L_{1 q}-L_{1 q}} n_{2} \\
K_{54} & =\frac{L_{a q s}^{\prime \prime} L_{1 q}}{L_{a q s}^{\prime \prime}-L_{a q s}^{\prime \prime} n_{4} L_{1 q}-L_{1 q}} n_{3} \\
K_{56} & =\frac{L_{a q s}^{\prime \prime} L_{1 q}}{L_{a q s}^{\prime \prime}-L_{a q s}^{\prime \prime} n_{4} L_{1 q}-L_{1 q}}\left(n_{5}-\frac{1}{L_{2 q}}\right) \\
K_{3}^{T C S C} & =\frac{L_{a q s}^{\prime \prime} L_{1 q}}{L_{a q s}^{\prime \prime}-L_{a q s}^{\prime \prime} n_{4} L_{1 q}-L_{1 q}} n_{6}
\end{aligned}
$$


Constants for $\Delta \psi_{2 q}$

$$
\begin{aligned}
T_{66} & =\frac{L_{2 q}^{2}}{\omega_{0} R_{2 q}\left(L_{a q s}^{\prime \prime} n_{5} L_{2 q}+L_{2 q}-L_{a q s}^{\prime \prime}\right)} \\
K_{62} & =\frac{L_{a q s}^{\prime \prime} L_{2 q}}{L_{a q s}^{\prime \prime}-L_{a q s}^{\prime \prime} n_{5} L_{2 q}-L_{2 q}} n_{1} \\
K_{63} & =\frac{L_{a q s}^{\prime \prime} L_{2 q}}{L_{a q s}^{\prime \prime}-L_{a q s}^{\prime \prime} n_{5} L_{2 q}-L_{2 q}} n_{2} \\
K_{64} & =\frac{L_{a q s}^{\prime \prime} L_{2 q}}{L_{a q s}^{\prime \prime}-L_{a q s}^{\prime \prime} n_{5} L_{2 q}-L_{2 q}} n_{3} \\
K_{65} & =\frac{L_{a q s}^{\prime \prime} L_{2 q}}{L_{a q s}^{\prime \prime}-L_{a q s}^{\prime \prime} n_{5} L_{2 q}-L_{2 q}}\left(n_{4}-\frac{1}{L_{1 q}}\right) \\
K_{4}^{T C S C} & =\frac{L_{a q s}^{\prime \prime} L_{2 q}}{L_{a q s}^{\prime \prime}-L_{a q s}^{\prime \prime} n_{5} L_{2 q}-L_{2 q}} n_{6}
\end{aligned}
$$

The expressions for $n_{1}-n_{6}$ and $m_{1}-m_{6}$ can be found in [2].

\section{APPENDIX B \\ SYSTEM DATA}

Generator parameters in pu:

$$
\begin{array}{llll}
x_{d}=1.81 & x_{q}=1.76 & x_{l}=0.16 & R_{a}=0.003 \\
L_{f d}=0.153 & R_{f d}=0.0006 & L_{1 d}=0.14 & R_{1 d}=0.0248 \\
L_{1 q}=0.7063 & R_{1 q}=0.0061 & L_{2 q}=0.1102 & R_{2 q}=0.0227 \\
x_{d}^{\prime}=0.65 & x_{d}^{\prime \prime}=0.23 & x_{q}^{\prime \prime}=0.25 & T_{q 0}^{\prime}=1.0 s \\
T_{d 0}^{\prime \prime}=0.03 s & T_{q 0}^{\prime \prime}=0.07 s & A_{\text {sat }}=0.031 & B_{\text {sat }}=6.93 \\
S T_{1}=0.8 & H=3.5 & K_{D}=0 & P_{t 0}=0.45
\end{array}
$$

$Q_{t 0}=0.15$

Exciter parameters:

$$
\begin{array}{llll}
K_{A}=0.1 & T_{A}=1 & T_{B}=10 & T_{R}=0.01 \\
K_{S T A B}=20 & T_{w}^{\prime}=10 & T_{1}^{\prime}=0.05 & T_{2}^{\prime}=0.02 \\
T_{3}^{\prime}=3 & T_{4}^{\prime}=5.4 & &
\end{array}
$$

TCSC and network data:

$$
\begin{array}{lll}
E_{B}=1 & R_{E}=0 & X_{E}=0.0025(\mathrm{pu}) \cdot L(\mathrm{~km}) \\
X_{T C S C}^{0}=10 \% \cdot X_{E} & T_{w}=1.5 \mathrm{~s} & T_{\text {local }}=0.05 \mathrm{~s} \\
T_{\text {remote }}=0.2 \mathrm{~s} & &
\end{array}
$$

\section{ACKNOWLEDGMENT}

Nguyen Tuan Anh would like to thank the Belgian Technical Cooperation for granting him a scholarship to carry out his $\mathrm{PhD}$ studies at the KU Leuven, Belgium.

\section{REFERENCES}

[1] M. Klein, G. J. Roger and P. Kundur, "A fundamental study of inter-area oscillations", IEEE trans. on power syst., Vol. 6, No.3, Aug. 1991, pp. 914-921.

[2] P. Kundur, Power System stability and Control, New York: McGraw-Hill, 1994.
[3] M. E. Aboul-Ela, A. A. Sallam, J. D. McCalley and A. A. Fouad, "Damping Controller Design for Power System Oscillations Using Global Signals," IEEE trans. on power syst., Vol. 11, No. 2, May 1996, pp. 767 773.

[4] N. G. Hingorani and L. Gyugyi, Understanding FACTS: Concepts and Technology of Flexible AC Transmission Systems, IEEE press, New York, 2000.

[5] R. M. Mathur and R. K. Varma, Thyristor-based FACTS controllers for electrical transmission systems, IEEE Press, Wiley-Interscience, 2002.

[6] Nguyen Tuan A., Van Hertem D. and Driesen J., "Effectiveness of TCSC controllers using remote input signals for transient stability enhancement," IEEE PES PowerTech, June 2011, pp. 1-8.

[7] X. Xie et al., "WAMS application in Chinese power system," IEEE Power Energy Mag., vol. 4, no. 1, pp. 54-63, Jan. 2006.

[8] I. C. Decker, D. Dotta, M. N. Agostini, S. L. Zimath and A. S. Silva, "Performance of a synchronized phasor measurements system in the Brazilian power system," Proc. IEEE Power Eng. Soc. General Meeting, Montreal, QC, Canada, 2006.

[9] F. L. Pagola, I. J. Perez-Arriaga and G. C. Verghese, "On sensitivities, residues and participations: applications to oscillatory stability analysis and control," IEEE trans. on power syst., Vol. 4, No. 1, Feb. 1989, pp. 278-285.

[10] L. Rouco and P. L. Pagola, "An eigenvalue sensitivity approach to location and controller design of controllable series capacitors for damping power system oscillations," IEEE trans. on power syst., Vol. 12, No.4, Nov. 1997, pp. 1660-1666.

[11] N. Yang, Q. Liu and J. D. MacCalley, "TCSC controller design for damping interarea oscillations," IEEE trans. on power syst., Vol. 13, No. 4, Nov. 1998, pp. 1304-1310.

[12] The Electric Power Research Institute (EPRI) WO 3789-05, ”Assessment of application and benefits of phasor measurement technology in power systems," Final Report by General Electric (GE) Company, EPRI Report TR-107903, April 1997.

[13] R. K. Varma, R. P. Guptay, and S. Auddyz, "Damping of inter-area oscillation in power systems by static var compensator (SVC) using PMUacquired remote bus voltage angles," International Journal of Emerging Electric Power Systems, Vol. 8, 2007.

[14] C. W. Taylor, M. V. Venkatasubramanian, and Y. H. Chen, "Wide-area stability and voltage control," In Proc. of the VII Symposium of Specialist in Electric Operational and Expansion Planning, 2000.

[15] A.G. Phadke and J.S. Thorp, Synchronized Phasor Measurements and their Applications, New York: Springer Science + Business Media, LLC, 2008. 